\title{
Foreword
}

\section{The Early Detection Research Network Second Annual Scientific Workshop 14-16 October 2001, Seattle, Washington, USA}

\author{
Sudhir Srivastava \\ Cancer Biomarkers Research Group, Division of \\ Cancer Prevention, National Cancer Institute, \\ National Institutes of Health, Bethesda, MD 20892, \\ USA
}

Studies have shown that detection of early cancer can reduce morbidity and mortality, for example, from mammography and Pap screening. Nevertheless, even these established technologies are limited by suboptimal sensitivity and specificity. Therefore, it seems reasonable to explore the application of the new molecularbased technologies for earlier and for more specific detection and even for assessment of risk, that is, before cancer physically develops, in order to institute prevention.

The application of molecular methods in the field of early cancer detection is a dynamic process continually driven by expanding knowledge of carcinogenesis and limited by the availability of technologies. At the molecular level, the detection of earlier cancer will require a more complete understanding of the evolution of preneoplastic lesions and associated molecular and genetic changes, evaluation and development of noninvasive detection technologies suited to incipient neoplastic lesions, and eventually identification of translational research to accelerate the identification of cancer at the earliest stages. There is a need to rapidly identify gene deletions, gene amplifications, or expression patterns in pre-malignant lesions and early cancers with high risk of progression so that cancer treatment or prevention can be more appropriately targeted and improved. We must also learn to recognize those lesions that would spontaneously regress and do not require aggressive treatment. Therefore, it is essential to develop a systematic and integrated research to promote molecular targets-based translational research for early detection in clinical settings.

The challenges of earlier cancer detection are multidisciplinary in nature. There are many issues at the level of basic science, the development of technology, the finding of key genetic changes, and the statistical analysis of changes in multiple gene functions often associated with the translation of laboratory findings into clinical application. A primary strategy is to facilitate an ongoing interaction between basic scientists, oncologists, clinicians, pathologists, geneticists, theoretical and applied biostatisticians, epidemiologists and other health professionals, since this is critical for the successful application of new discoveries in molecular biology for earlier detection. The National Cancer Institute initiated a major program "The Early Detection Research Network" to address discovery and application of molecular markers in earlier cancer detection and risk assessment. The current issue of Disease Markers presents the abstracts presented at the Second Annual Conference of this Network, held at Seattle, Washington. The abstracts cover a wide range of topics relevant to the field of cancer detection and cancer risk assessment. 


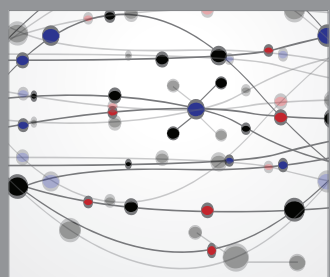

The Scientific World Journal
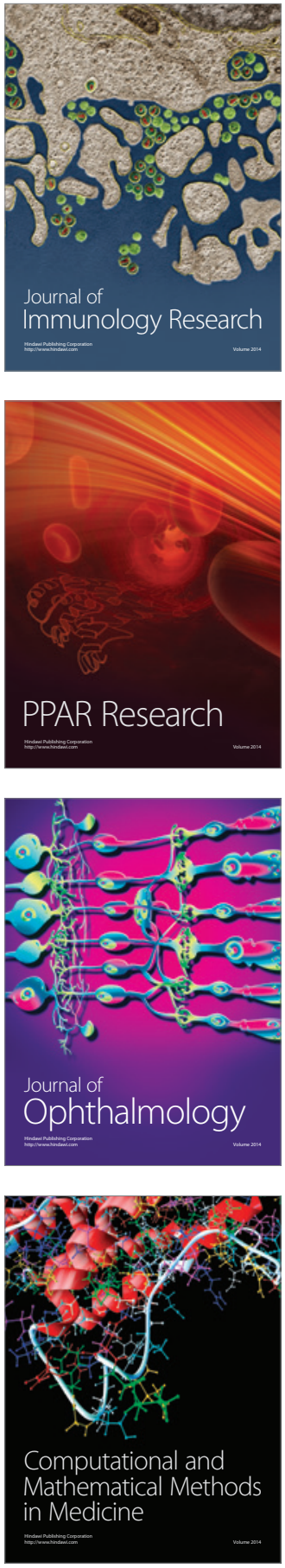

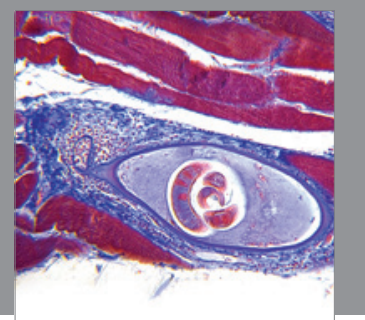

Gastroenterology

Research and Practice
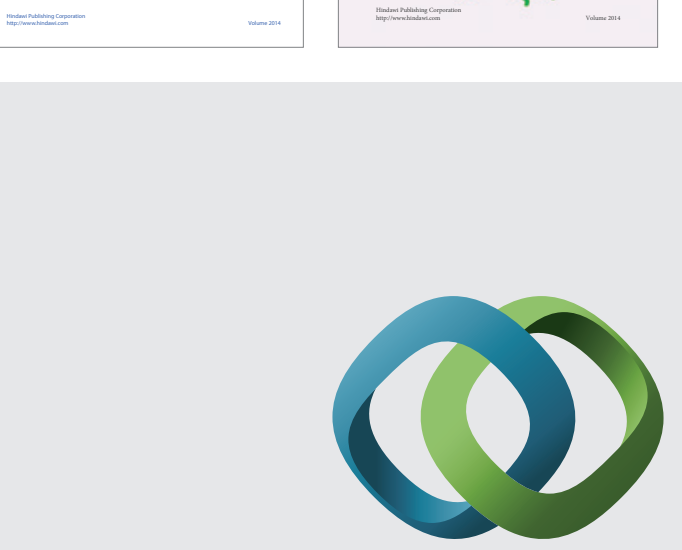

\section{Hindawi}

Submit your manuscripts at

http://www.hindawi.com
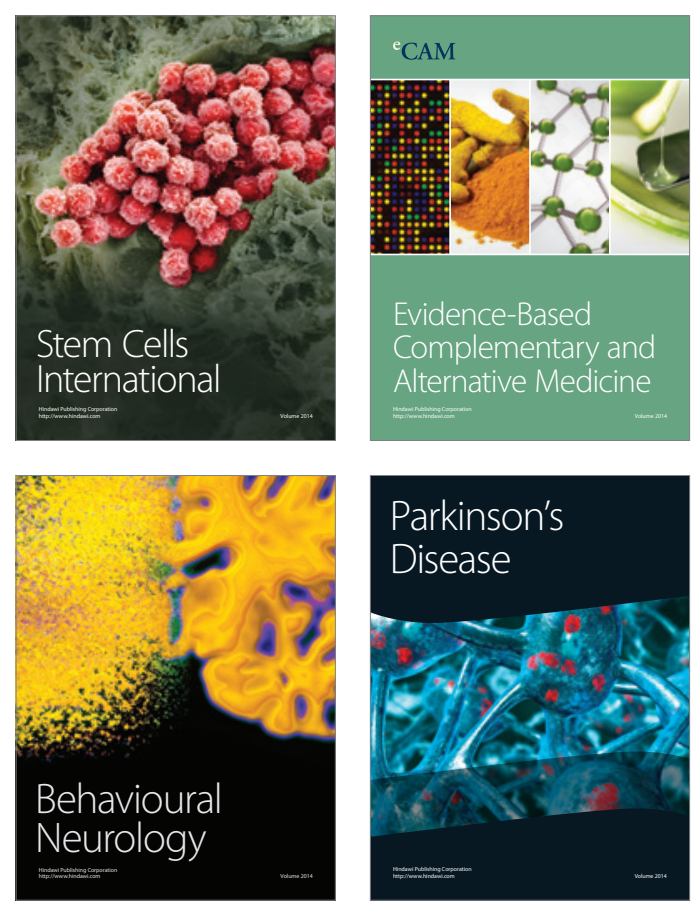

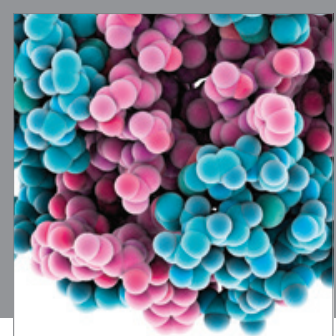

Journal of
Diabetes Research

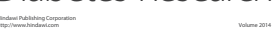

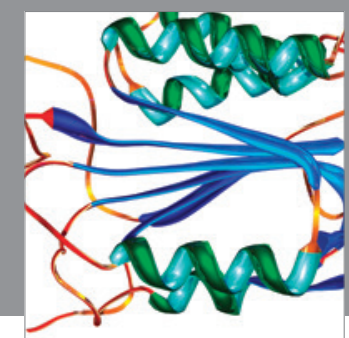

Disease Markers
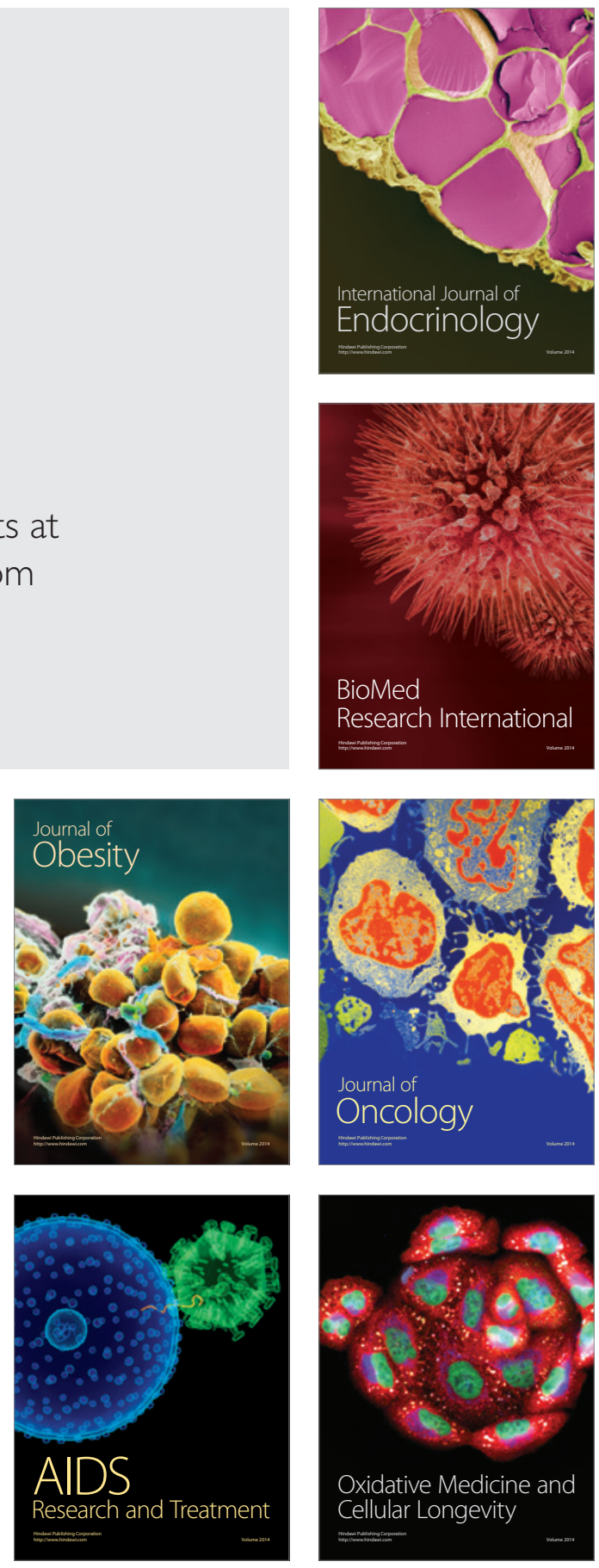